\title{
On the origin of the observer
}

\author{
Amrit Sorli \\ Foundations of Physics Research Centre - FoPRC, via Resistenza 1087053 Celico (CS), Italy
}

Email address:

sorli@foprc.org

To cite this article:

Amrit Sorli. On the Origin of the Observer. American Journal of Modern Physics. Vol. 3, No. 4, 2014, pp. 173-177. doi: 10.11648/j.ajmp.20140304.14

\begin{abstract}
Observer is a fundamental element of physics and science in general. It also plays an important role in modern psychology as an entity that has ability to be aware of all different contents of conscious and unconscious mind. Observer is a central point of developed personality and represents the origin of creativity, auto-reflective thinking and mental health. Until one is aware of his mind activity which might be fully destructive, he will not go insane. Human mind changes continuously, observer is always the same, stable and present. In generally in the universe all that is made out of quanta changes and is in motion. From this perspective the origin of the observer might be beyond known matter and energy in the sense of Einstein's formula $E=m c 2$. Trivalent logic allows existence of some entity that is not A (matter), is not B (energy), but is C. Application of bijective epistemology and theory of numbers confirm observer might have non-material origin.
\end{abstract}

Keywords: Observer, Being, Observing, Watching, Bijective Epistemology, Set Theory, Theory of Numbers, Trivalent Logic, Theory of Everything, Self-Realization

\section{Introduction}

In 1935 Einstein, Podolski and Rosen (EPR) introduced a completeness criterion for quantum mechanics. It is stipulated that a necessary requirement for a complete theory is that "every element of physical reality must have a counterpart in the physical theory. The elements of the physical reality cannot be determined bya priori philosophical considerations, but must be found by an appeal to results of experiments and measurements" [1].

In order to satisfy completeness criterion I introduce Bijective epistemology founded on bijective function of set theory according to which a given element in set $\mathrm{X}$ corresponds exactly one element in the set Y:

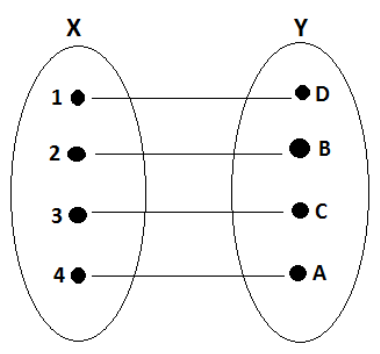

Figure 1. A bijective function, $f: X \rightarrow Y$, where set $X$ is $\{1,2,3,4\}$ and set $Y$ is $\{A, B, C, D\}$. For example, $f(1)=D$.
Within Bijective Epistemology, each element in a model of phenomenon we are considering exactly corresponds to the one and only one element of phenomenon itself. In this way, a model becomes an adequate picture of a given phenomenon, between model representing this phenomenon and phenomenon itself being a direct epistemological correlation.

In Bijective Epistemology each element of the phenomenon under examination needs to be firstly perceived and then can be considered in existence. In natural sciences a given element is perceived by outer senses. In human sciences a given element can be perceived also by inner sense as for example thoughts, emotions, sensations, etc.

Let's apply bijective epistemology to the Theory of Everything (TOE). In a physical Universe the following five elements are perceived by senses: matter, energy (all types of electromagnetic energy), space, change and time as duration of changes, running in space. These are perceivable elements of set »universe $\mathrm{X} \ll$. The sixth element of the set universe $\mathrm{X}$ is the observer, which perceives the other five elements. Using a bijective function, we can transform these six elements of the set universe $\mathrm{X}$ into six elements of model set »model $\mathrm{Y} «$ as shown in Figure 2 [2]. 


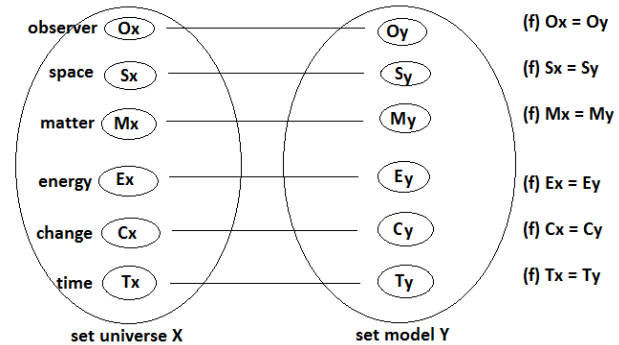

Figure 2. Between set universe $X$ and set model $Y$ there is a direct epistemological co-relation

Observer has experimental evidence that matter can transform into energy and that matter is composed by energy. Furthermore, particles spontaneously appear from space and disappear back in it, leading to the assumption that also space is a type of energy (named quantum vacuum). Space, matter and electromagnetic energy are different types of "energy".

\section{On the Origin of the Observer}

The above model shows that observer and energy are two different elements. One cannot exclude that observer is not made out of energy as known in today physics. Trivalent logic [3] allows existence of an entity which is neither matter nor energy. It is difficult to imagine, in the framework of a modern science, what this could be but we cannot exclude existence of phenomena which are not a form of energy.
The master mathematician and physicist Carl Friedrich Gauss (1777-1855) said that "Mathematics is the Queen of the Sciences and Arithmetic the Queen of Mathematics" [4]. I will explore in this paper the origin of observer on the basics of arithmetic's and set theory.

On the basis of our daily experience we can conclude that there is no observer in us as an entity which is observing. We are observing (watching) outer and inner world and we are aware of observation itself. Behind observation, there is no "I'm", on the contrary, there are all different aspects of "I'm" which we can observe and be aware of. This experience indicates that there is no observer "as such" (in Kantian sense), observation is a function of our true self, we can also name it "Being", called "Emptiness" in eastern mysticism.

Being-Emptiness exists but it has no physical attributes of matter and energy. In Arithmetic Emptiness corresponds to the number zero. In set theory number zero is empty set. In this picture number 1 represents energy of quantum vacuum which in today physics is as the fundamental source of all different types of energy. Number 2 represents electromagnetism and number 3 represents elementary particles:

$0 \equiv \emptyset=\{\}$ (empty set) represents Emptiness-Being

$1 \equiv 0^{\prime}=\{0\}=\{\varnothing\}$ represents quantum vacuum

$2 \equiv 1^{\prime}=\{0,1\}=\{0,\{0\}\},=\{\varnothing,\{\varnothing\}\}$ represents electromagnetic energy

$3 \equiv 2^{\prime}=\{0,1,2\}=\{0,\{0\},\{0,\{0\}\}\}=\{\varnothing,\{\varnothing,\{\varnothing$, $\{\varnothing\}\}\}\}$ represents elementary particles.

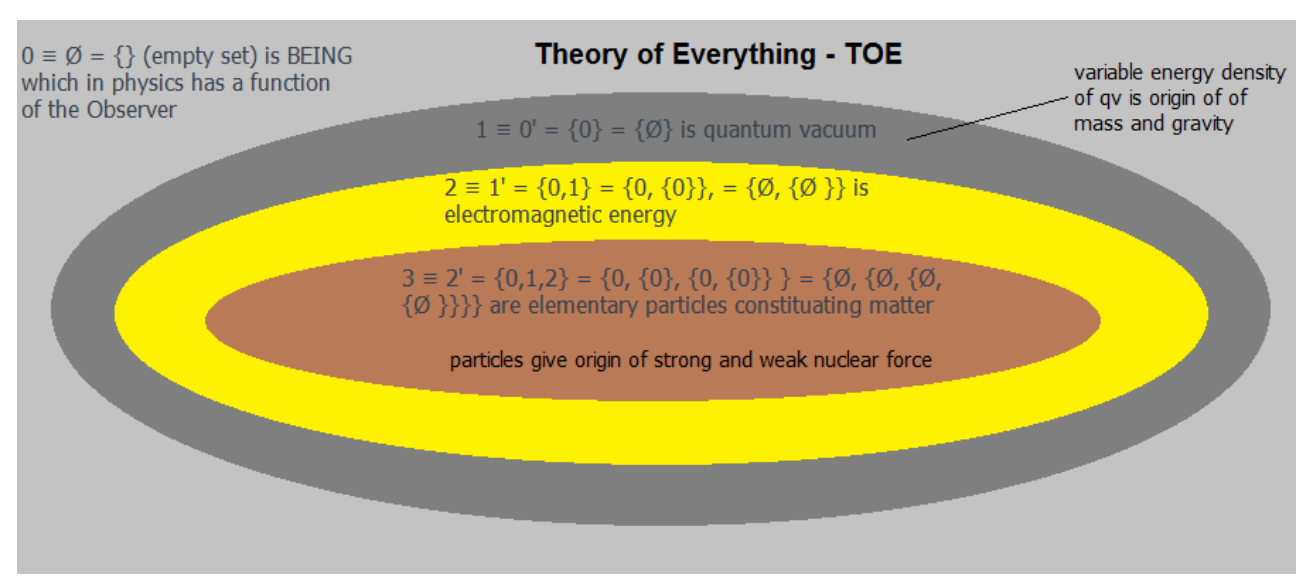

Figure 3. Theory of Everything derived from set theory

\section{Discussion}

In this picture of Theory of Everything we have only two existential numbers: 0 and 1 . Number 2 is already a manifestation of number 1 and number 3 is manifestation of number 2. It is interesting that all the computers work on mathematical model which has only number 0 and number 1. All the other numbers can be derived from these two numbers. An ancient proverb says: "Mathematics is a God face mirroring in the human mind". It seems mathematic reflects some fundamental laws of the universe.
The consideration that the observer has no physical origin is an important standpoint which allows human being to discover realms of reality which are beyond standard reductionist picture of western science. How Being is acting on the physical level is not discovered yet fully, modern physics gives a basic model represented in figure 4. Being is connected with physical world via quantum vacuum and electromagnetic energy (bio-photons) [5] as carriers of its action. Regarding observationwatching as its fundamental function in the human organism the Being is acting as follows on figure 4 below: 


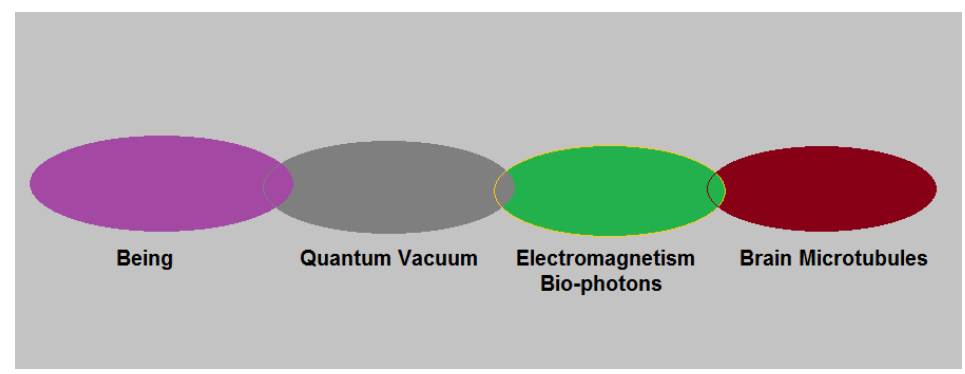

Figure 4. Acting of Being

Being acts via quantum vacuum and bio-photons on brain microtubules which are related to the senses with nervous system. In this model consciousness does not exist on its own: to observe something and to be conscious about it is a function of Being. To be conscious of some object which might be material or psychological (thought, emotion, self-image, memory, scientific model of material world) implies a "process" of knowing which has three stages: perception of an object through senses or through inner perception, experience of an object and finally awareness of the object existence. In the process of knowing we have a "subject" which is conscious about an "object". Being has an inherent self-recognition which is not a "process"; Being is not changing, is not a process and is not made out of "quanta" which are under the rule of change. However Being acts in material world via quantum vacuum which is energy in its original state. In the model of Theory of everything (TOE) here presented universe has three levels:

- Being is non-material level which is nonlocal

- quantum vacuum is a fundamental state of energy which is nonlocal

- $\quad$ quanta (photon and elementary particles building matter) are different forms of quantum vacuum energy) are local

In this model human mind is an unknown undiscovered yet form of quantum vacuum energy (different from known forms of quanta) which is "software" and human brain which is "hardware" of human organism. From this perspective consciousness as a function of Being has physical origin in quanta which are special forms of quantum vacuum similarly as human mind. Between Being and senses they are following elements:

- Being

- Consciousness as a function of Being made out of special form of quanta

- $\quad$ Mind made out of special form of quanta

- Bio-photons

- Brain microtubules

- Nervous system

- Senses.

This view on consciousness and its relation to the human organism is close to "quantum theory of consciousness" of Hameroff and Penrose where consciousness is a nonlocal phenomenon not supposed to have physical origin in the human brain. [6].

Considering human mind is a special form of nonlocal quantum vacuum one can predict human mind can communicate with other human mind instantly (telepathy) via quantum vacuum without using senses. Research of our research group shows universal space which originates from quantum vacuum is a medium of immediate information transfer between quanta in EPR experiment [7] Further on variable energy density of quantum vacuum gives origin to the inertial mass and to the gravitational mass [8]. In our model quantum vacuum is a dynamic medium of energy in its original state which plays essential role in universal dynamics, functioning of living organism and evolution of life as well. Dynamic nonlocal quantum vacuum is timeless in a sense time in quantum vacuum exists only as a mathematical parameter of change.

\section{Awakened Observer is aware of inner psychological Time "Past-present- Future"}

According to "Second attention epistemology" research, the common observer in its daily life is only partly aware of how his mind elaboration influences his experience. He experiences world and relations with other humans mostly through his emotions and memories. There is an information gap between perception which happens in senses and experience [9]. When observer is fully merged in Being, which happen when it is one with the Zero \{\} (empty set) his experience is "free" of any mind interpretation. Awakened observer experiences directly what senses perceive. His experience is "objective" in a sense that there is no "subjective" interpretation of the mind.

Our research on the subject of time confirms in this direct experience, awakened observer experiences that time we measure with clocks is only duration of change which run in timeless quantum vacuum in "eternal now and here". This duration has its internal numerical order, namely change $X+1$ is after change $X$, change $X+2$ is after change $\mathrm{X}+1$, but all changes run in space which is timeless by itself. Numerical order of change is fundamental time, duration of change is emergent time gained by measurement. Fundamental unit of numerical order is 
Planck time $t_{P n}$. Duration $t$ is the sum of numerical order: $t=t_{P, 1}+t_{P, 2}+\ldots+\mathrm{t}_{P, N}=\sum_{i=1}^{N} t_{P, i}[10]$.

Our research confirms time we measure with clocks is a fundamental quantity of physics which has exclusively a mathematical existence. Changes run in space only and not in time. Time is not $4^{\text {th }}$ dimension of space and does not flow in space by itself as a physical reality in which changes run. [10]. From this perspective "arrow of time" has only a mathematical existence pointing in the direction of numerical order flow: $t_{P, 1} \rightarrow t_{P, 2} \ldots \rightarrow \mathrm{t}_{P, N}$.

Awakened observer becomes fully aware that linear time "past-present-future" is a psychological time which exists only in the mind, in physical universe the only time is Einstein's NOW: "...there is something essential about the NOW which is just outside the realm of science. People like us, who believe in physics, know that the distinction between the past, present and future is only a stubbornly persistent illusion". Albert Einstein [11].

Western scientific mind explores the Universe and life only within the framework of linear psychological time »past-present-future $\ll$. A sensation of duration is a consequence of our experiencing the changes within the framework of psychological time. In physics, the duration of a given change enters into existence when it is being measured. There is no duration without a measurement that is done by the observer. For the observer who is experiencing within the framework of psychological time this duration is "real", it has an existence of its own. For the awakened observer which is free of psychological time duration, it is only a by-product of the act of measurement, it has no independent existence. Changes in the universe, nature, and our lives simply take place in the $N O W$ which is eternity itself. Awakened observer distinguishes between fundamental time which is a numerical order of change, emergent time which is duration of change and psychological time which is a mind model. Physics enriched with awakened observer will encourage the development of a integral science within which the surveys of both the material world and of the Being will be balanced.

\section{Self-Realization as a Process of Elimination}

Being is the source of "awakened observer" which is fully aware how his mind (thoughts and emotions, memories) influences his experience. Awakened observer free of any psychological bondage realizes that whatsoever he can watch (witness) is not him. You can become conscious of all "material and psychological objects" which you are not: your house, your family members, your friends, your body, your emotions, your self-images, your memories and finally your fear of death. You cannot become conscious about yourself because you are not an object. When you "eliminate" all objects you are conscious of them what remains is "You". And this "You" is unborn, immortal and far beyond of death of your physical and mental body. In Indian Advaita Vedanta this technique in which you "eliminate" all objects you are conscious of them, is called "Neti Neti"; it means "neither this, nor that" [12].

Self-realization is a »transcendental jump « from 1 to 0 . It is far beyond rational human mind comprehension. In set theory we understand that number 1 is an empty set with element 0, however how exactly Being appears in manifested physical world remain a secret. Research on observer and Being requires "experiential approach". You can understand how 1 emerges from 0 and still you do not know 0 . Experiencing 0 is purely experiential by each individual and reaches beyond rational mind.

\section{Conclusions}

In modern science observer is still a substantially unexplored subject. Exploration of observer reaches far beyond established procedure of "objective" scientific research because observation is a not objective reality as for example an atom, observation is a subjective phenomena. To the question "Who is the observer?" the best answer is that it is the rational mind which has raised the question. The answer is not intellectual, it is experiential. The above presented model is only a picture of reality and not the true reality of the observer itself. It gives only a few hints which may help someone to start experimental research on the observer which is observing-watching the mind. The bridge between number 0 (non-manifested) and number 1 (manifested) is a mystery that one cannot fully understand by rational mind. Observing (watching, witnessing) how rational mind function and becoming aware you are not the mind is the only path to the Being.

\section{Acknowledgement}

The author wish to thank Luigi Maxmilian Caliguiri and Pier Luigi Lattuada for their advices and encouragement.

\section{References}

[1] A. Einstein, B. Podolski and N. Rosen, "Can the quantummechanical description of physical reality be considered complete?", Physical Review, 1935.

[2] Luigi Maxmilian Caligiuri, Amrit Sorli. Special Theory of Relativity Postulated on Homogeneity of Space and Time and on Relativity Principle. American Journal of Modern Physics. Vol. 2, No. 6, 2013, pp. 375-382. doi: 10.11648/j.ajmp.20130206.25

[3] J. Łukasiewicz, O logice trójwartościowej (in Polish,1920). Ruch filozoficzny 5,170. English translation: On threevalued logic, in L. Borkowski (ed.), Selected works by Jan Eukasiewicz (North-Holland, Amsterdam, 1970), pp. 87-88. 
[4] Edna E. Kramer, The Nature and Growth of Modern Mathematics, Princeton university press (1970)

[5] Popp, F. A., Chang J.J., Herzog, A., Yan, Z., Yan, Y. (2002) Evidence of non-classical (squeezed) light in biological systems. Physics Letters A, 293(1-2): 98-102.

[6] Hameroff S., Penrose R. Consciousness in the universe - A review of the 'Orch OR' theory, Physics of Life Reviews 11:39-78 (2014), http://www.journals.elsevier.com/physicsof-life-reviews/

[7] Fiscaletti D., Sorli A., Three-dimensional space as a medium of quantum entanglement, Annales UMSC Physica, Vol. 67, Issue 1 (2012), DOI: 10.2478/v10246-012-0014-5

[8] Luigi Maxmilian Caligiuri, Amrit Sorli. Gravity Originates from Variable Energy Density of Quantum Vacuum. American Journal of Modern Physics. Vol. 3, No. 3, 2014, pp. 118-128. DOI: 10.11648/j.ajmp.20140303.11

[9] Lattuada P. ,»Second Attention Epistemology: Truth and Reality«, Integral Transpersonal Journal, Number 0 (2010)

[10] Amrit Sorli, Dusan Klinar, and Davide Fiscaletti. "New Insights into the Special Theory of Relativity." Physics Essays, 24, 2 (2011) http://dx.doi.org/10.4006/0836-139826.1.113

[11] Amrit Sorli. The Physics of NOW, Amazon (2013)

[12] Vishnu Devananda. Meditation and Mantras: An Authoritative Text. New Delhi: Motilal Banarsidass Publishers. p.119. (1999) 